\title{
Fuzzy Systems for Multicriteria Decision Making
}

\author{
Fábio J. J. Santos \\ Federal Institute of Education, Science and Technology of São Paulo (IFSP) and Federal \\ University of São Carlos (UFSCar) \\ Araraquara, SP, Brazil, \\ fabio_santos@dc.ufscar.br \\ and

Heloisa A. Camargo
Computer Department - Federal University of São Carlos (UFSCar)
São Carlos, SP, Brazil,
heloisa@dc.ufscar.br

\begin{abstract}
One of the techniques used to support decisions in uncertain environments is the Fuzzy TOPSIS method. However, from crisp data, this method considers only one fuzzy set in their analysis, besides being a strictly mathematical optimization technique. This article proposes extensions to the original Fuzzy TOPSIS, exploring two distinct versions: to increase the method with the necessary resources for the mathematics process to consider the membership values of the input data in more than one fuzzy set and to aggregate to method the empiric knowledge of an expert represented through fuzzy rules. In such case, the method, named by Fuzzy F-TOPSIS (Fuzzy Flexible TOPSIS), is proposed with the objective of improving the Fuzzy TOPSIS ability to deal with uncertainty through the combination of the mathematical process involved in the original Fuzzy TOPSIS with the expert empirical knowledge. A case study is presented to validate the proposal.
\end{abstract}

Keywords: Fuzzy TOPSIS, Fuzzy Rule-Based Systems, Decision Support Systems, Multicriteria Decision Making

\section{Introduction}

The use of fuzzy logic and of fuzzy sets theory applied to the decision making process was proposed initially in [1]. Since then, several works have been conducted aiming to support the decision making process. Some Decision Support Systems (DSS) are based on classical techniques for decision making as, for example, Decision Tree [2], [3], [4], e [5]. In multicriteria optimization problems, one of the methods that has shown growth in the field of research is the TOPSIS (Technique for Order Preference by Similarity to Ideal Solution). Initially proposed by Hwang \& Yoon [1] to treat problems with numerical values, currently the technique has many applications and contributions to optimization and manipulation of inaccurate data by Fuzzy TOPSIS [2, 3, 4].

Fuzzy TOPSIS model, proposed in [4], allows the values and weights of the attributes are defined using fuzzy numbers instead of precise numbers. However, this flexibility is applied only in the definition of values. The process of analysis and recommendation of the best alternative is based on conventional optimization techniques. Other characteristic of the method presented in [4], is that the linguistic value considered in the process will be only the fuzzy set of greater pertinence for the input numeric value.

Thus, to improve the ability of deal with vagueness in Fuzzy TOPSIS is proposed the Fuzzy F-TOPSIS (Fuzzy Flexible TOPSIS), which has as its main contributions the aggregation of Fuzzy Rule-Based Systems to Fuzzy TOPSIS, and the proposal of a mathematical model to take into account more than a fuzzy set from the input numeric value.

In Section 2, it is presented the basic concepts of fuzzy sets. The Fuzzy TOPSIS is presented in Section 3. The proposed method in this paper, as an extension to the Fuzzy TOPSIS, is described in Section 4. In Section 5, a case study in the CRM (Customer Relationship Management) area has been realized using the methods described and, 
also in the Section 5, a comparative analysis of the results is performed. Finally, the conclusions are presented in Section 6.

\section{Fuzzy Sets Theory}

The fuzzy sets are represented by linguistic terms that builds one or more linguistic variables, ie, the linguistic variables have their possible states defined in a universe of discourse $U$, represented by these linguistic terms.

A fuzzy set $A$, in a universe of discourse $U$, is characterized by a pertinence function $A \rightarrow[0,1]$, where $A(x), \forall x \in$ $U$, indicates the membership degree of $x$ in $A[6]$.

The representation of fuzzy sets can be done in many ways as, for example, triangular, trapezoidal or gaussian. The context of situation will determine what is the best among the existing ones. In this paper we use the representation by triangular fuzzy sets.

A triangular fuzzy set $A$ has its membership function defined by Equation (1) and can be denoted by $\left(a_{1}, a_{2}, a_{3}\right)$.

$$
A(x)=\left\{\begin{aligned}
0, & \text { se } x \leq \mathrm{a}_{1} \\
\frac{x-\mathrm{a}_{1}}{\mathrm{a}_{2}-\mathrm{a}_{1}}, & \text { se } x \in\left(\mathrm{a}_{1}, \mathrm{a}_{2}\right] \\
\frac{\mathrm{a}_{3}-x}{\mathrm{a}_{3}-\mathrm{a}_{2}}, & \text { se } x \in\left(\mathrm{a}_{2}, \mathrm{a}_{3}\right) \\
0, & \text { se } x \geq \mathrm{a}_{3}
\end{aligned}\right.
$$

Given $\tilde{A}$ and $\tilde{B}$ two triangular fuzzy sets defined as $\left(a_{1}, a_{2}, a_{3}\right)$ and $\left(b_{1}, b_{2}, b_{3}\right)$ respectively, the multiplication operation in these sets is as follows:

$$
\tilde{A}(\times) \tilde{B}=\left(a_{1}, a_{2}, a_{3}\right)(\times)\left(b_{1}, b_{2}, b_{3}\right)=\left(a_{1} b_{1}, a_{2} b_{2}, a_{3} b_{3}\right)
$$

According to [7], the distance between two triangular fuzzy sets can be calculated by Equation (3).

$$
d(\tilde{A}, \tilde{B})=\sqrt{\frac{1}{3}\left[\left(a_{1}-b_{1}\right)^{2}+\left(a_{2}-b_{2}\right)^{2}+\left(a_{3}-b_{3}\right)^{2}\right]}
$$

Given the fuzzy sets $\tilde{A}, \tilde{B}$ and $\tilde{C}$, the fuzzy set $\tilde{B}$ is closer to $\tilde{A}$ than $\tilde{C}$ if, and only if, $d(\tilde{A}, \tilde{B})<d(\tilde{A}, \tilde{C})$.

\section{The Fuzzy TOPSIS Method}

The multicriteria decision making problem with fuzzy sets was first introduced by Zadeh and Bellman in [8]. The essence of this class of problems is the processing of information in order to consider different attributes with different importance degrees in the process for proposing a solution to the problem analyzed. The weight of these attributes can be defined empirically or using techniques as AHP (Analytic Hierarchy Process) [9] and [10] or the ANP (Analytic Network Process) [11] and [12].

The Fuzzy TOPSIS method is based on a procedure that, after the initial steps to calculate the decision matrix, the Positive Ideal Solution (PIS) and Negative Ideal Solution (NIS) are defined. The first, PIS, aims to maximize the benefits attributes and minimize the costs attributes. However, the NIS minimizes the benefits attributes and maximizes the costs attributes of a possible solution to the problem. Among the alternatives available, the alternative that is closer to the PIS and farther from the NIS will be defined as the best solution.

In the Fuzzy TOPSIS method a MCDM (Multi-Criteria Decision-Making) problem with $m$ alternatives $\left(A_{1}, A_{2}, A_{3}\right.$, $\left.\ldots, A_{m}\right)$ and $n$ attributes $\left(C_{1}, C_{2}, C_{3}, \ldots, C_{n}\right)$ can be expressed in matrix format according to (4) and (5),

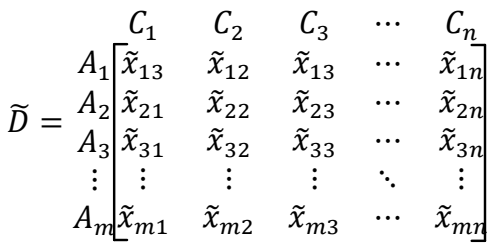




$$
\widetilde{W}=\left[\widetilde{w}_{1}, \widetilde{w}_{2}, \ldots, \widetilde{w}_{n}\right],
$$

where $\tilde{x}_{i j}, i=1,2, \ldots, m$, and $j=1,2, \ldots, n$ are numeric data of problem and $\widetilde{w}_{j}, j=1,2, \ldots, n$ is the importance degree of each attribute $C_{1}, C_{2}, \ldots, C_{n}$, respectively.

For the assignment of importance degree to the attributes, it will be used the empirical method adopted and described in [4], where is showed a scale from 1 (least important) to 5 (most important). From these values, the Table 1 shows the equivalence between the numeric values and the fuzzy sets used in this paper.

Table 1: Transformation for fuzzy membership functions

\begin{tabular}{lcl}
\hline Rank & Attribute grade & Membership functions \\
\hline Very Low (VL) & 1 & $(0.00,0.00,0.25)$ \\
Low (L) & 2 & $(0.00,0.25,0.50)$ \\
Medium (M) & 3 & $(0.25,0.50,0.75)$ \\
High (H) & 4 & $(0.50,0.75,1.00)$ \\
Very High (VH) & 5 & $(0.75,1.00,1.00)$ \\
\hline
\end{tabular}

Based on the decision matrix (4) the data are normalized by Equation (6) for the attributes that should be maximized and by Equation (7) for the attributes that should be minimized.

$$
\begin{aligned}
& r_{i j}=\frac{x_{i j}-\min \left\{x_{i j}\right\}}{\left[\max \left\{x_{i j}\right\}-\min \left\{x_{i j}\right\}\right]} \\
& r_{i j}=\frac{\max \left\{x_{i j}\right\}-x_{i j}}{\left[\max \left\{x_{i j}\right\}-\min \left\{x_{i j}\right\}\right]}
\end{aligned}
$$

Calculated $r_{i j}$ from normalized matrix, the fuzzification of this value will be made according the membership functions in Table 1. Defined the fuzzy matrix with the fuzzy sets $\tilde{v}_{i j}$ and $\widetilde{w}_{i}$, we must calculate the weighted matrix using Equation (2), taking into account the importance degree $\left(\widetilde{w}_{i}\right)$ of each attribute and the set fuzzy $\left(\tilde{v}_{i j}\right)$ with greater membership function that represents $r_{i j}$. Calculated the weighted by Equation (2) matrix, the Fuzzy Positive Ideal Solution (FPIS, $A^{+}$), which represents the compromise solution, and the Fuzzy Negative Ideal Solution (FNIS, $\left.A^{-}\right)$, which represents the worst possible solution, can be defined respectively as $\tilde{v}_{j}^{+}=(1,1,1)$ and $\tilde{v}_{j}^{-}=(0,0,0)$, for $j=1,2, \ldots, n[13]$, for triangular set fuzzy.

The Euclidean distance of each alternative to FPIS and the FNIS is given by Equations (8) and (9) respectively.

$$
\begin{gathered}
d_{i}^{+}=\sum_{j=1}^{n} d\left(\tilde{v}_{i j}, \tilde{v}_{j}^{+}\right), i=1,2, \ldots, m ; j=1,2, \ldots, n \\
d_{i}^{-}=\sum_{j=1}^{n} d\left(\tilde{v}_{i j}, \tilde{v}_{j}^{-}\right), i=1,2, \ldots, m ; j=1,2, \ldots, n
\end{gathered}
$$

After to calculate the Euclidian distance from fuzzy sets to the FPIS and FNIS, by Equations (8) and (9), the degree of similarity of each alternative with the FPIS is given by Equation (10).

$$
C C_{i}=\frac{d_{i}^{-}}{d_{i}^{+}+d_{i}^{-}}
$$

The ranking of the alternatives will present as the best solution the alternative with the highest value resulting from Equation (10), followed by others in descending order.

\section{The Fuzzy F-TOPSIS Method}

Originally, Fuzzy TOPSIS is an optimization technique that uses linguistic terms to evaluate the importance of attributes and their values. In order to improve their ability to deal with vagueness, in this paper we propose adding 
to the Fuzzy TOPSIS an attribute $\left(C_{n+1}\right)$ that represents the evaluation of a Fuzzy Rules Based System. The main purpose of this modification is to add the capabilities of fuzzy rules based systems to Fuzzy TOPSIS. Thus, the analysis by Fuzzy F-TOPSIS will allow the empirical knowledge of the expert, represented by fuzzy rules, also be considered in the decision making and optimization process, in addition to definitions of the membership functions of the fuzzy sets.

The use of optimization techniques associated with the empirical knowledge of experts, allows us a hybrid analysis of the multicriteria problems where the process of decision making requires the use of human sensitivity, which often can be expressed by a fuzzy rules base. Thus, the behavior of the system may have greater influence, or not, than the rules defined by the expert. The influence level of these rules will be defined by influence degree that the attribute $\left(C_{n+1}\right)$ will receive in the analysis of the problem.

In order to obtain a better representation in the ranking made by Fuzzy F-TOPSIS, some adjustments in the definition of FPIS and FNIS have been performed. Instead of using the definition described in section 3 of this article, the definition of FPIS and FNIS as described in [1], presented a classification that had greater acceptance by the experts during the experiments. The definitions of FPIS and FNIS are presented in Equations (11) and (12).

$$
\begin{aligned}
& A^{+}=\left(\tilde{v}_{1}^{+}, \tilde{v}_{2}^{+}, \ldots, \tilde{v}_{n}^{+}\right)=\left\{\left(\max \tilde{v}_{i j} \mid i=1,2, \ldots m\right), j=1,2, \ldots, n\right\}, \\
& A^{-}=\left(\tilde{v}_{1}^{-}, \tilde{v}_{2}^{-}, \ldots, \tilde{v}_{n}^{-}\right)=\left\{\left(\min \tilde{v}_{i j} \mid i=1,2, \ldots m\right), j=1,2, \ldots, n\right\}
\end{aligned}
$$

Among all the weighted fuzzy sets calculated by Equation (2), for each of $C_{n+1}$ attributes involved in the problem, the largest coordinated $a_{1}, a_{2}$ and $a_{3}$ will be considered in defining of the FPIS. Similarly, however for the lower values of the coordinates $a_{1}, a_{2}$ and $a_{3}$ of each attribute, the FNIS will be defined.

Another contribution of this paper is the proposal to consider two fuzzy sets with pertinence for the input value $r_{i j}$. For this, two fuzzy matrices for decision making will be mounted. The first matrix, $V_{1}$, with the fuzzy sets of greater value of membership function for each of $r_{i j}$ values and, the second, $V_{2}$, with the fuzzy sets of lower value of membership function.

The calculation of Euclidian distance between the fuzzy sets $\tilde{v}_{i j}$ of the matrix $V_{1}$ and $V_{2}$ and the FPIS and FNIS, should take into account the Influence Level $\left(I L_{\widetilde{V}_{i j}}\right)$ of $r_{i j}$ in each of the fuzzy sets that has pertinence. In the set that has greater pertinence, the calculation of $I L_{\widetilde{V}_{i j}}$ will be made by Equation (11) and the set of lower pertinence by Equation (12). Case $I L_{\tilde{v} 1_{i j}}=1$, then $I L_{\tilde{v} 2_{i j}}=0$.

$$
\begin{aligned}
I L_{\tilde{v} 1_{i j}} & =\frac{\mu \tilde{v} 1_{i j}\left(r_{i j}\right)}{\mu \tilde{v} 1_{i j}\left(r_{i j}\right)+\mu \tilde{v} 2_{i j}\left(r_{i j}\right)} \\
I L_{\tilde{v} 2_{i j}} & =\frac{\mu \tilde{v} 2_{i j}\left(r_{i j}\right)}{\mu \tilde{v} 1_{i j}\left(r_{i j}\right)+\mu \tilde{v} 2_{i j}\left(r_{i j}\right)}
\end{aligned}
$$

Thus, the calculations of distances from $v_{i j}$ to the FPIS and FNIS, considering the influence level of $v_{i j}$ in the matrix, are defined as follows:

$$
\begin{gathered}
d_{i}^{+}=\sum_{j=1}^{n} d\left(\tilde{v} 1_{i j}, \tilde{v}_{j}^{+}\right) \times I L_{\tilde{v} 1_{i j}}+\sum_{j=1}^{n} d\left(\tilde{v} 2_{i j}, \tilde{v}_{j}^{+}\right) \times I L_{\tilde{v} 2_{i j}} \\
d_{i}^{-}=\sum_{j=1}^{n} d\left(\tilde{v} 1_{i j}, \tilde{v}_{j}^{-}\right) \times I L_{\tilde{v} 1_{i j}}+\sum_{j=1}^{n} d\left(\tilde{v} 2_{i j}, \tilde{v}_{j}^{-}\right) \times I L_{\tilde{v} 2_{i j}}
\end{gathered}
$$

According to Equations (13) and (14), the similarity degree between the alternatives $A_{i}$ and FPIS take into account the distance of the sets $\tilde{v}_{i j}$ in $V_{1}$ and $V_{2}$ for the FPIS and FNIS.

Just as the Fuzzy TOPSIS, the ranking of the alternatives will present as the best solution the alternative with the highest value resulting from Equation (10), followed by others in descending order. 


\section{Case Study}

To validate the method described here has done a study focusing on CRM (Customer Relationship Management) systems in a transport company. The problem consists in to establish the best customer of the company taking into account the attributes Revenue $\left(C_{1}\right)$, Percentage of bills late more than 30 days $\left(C_{2}\right)$, Regularity of payment bills $\left(C_{3}\right)$, Total weight carried $\left(C_{4}\right)$, Amount of invoice by customer $\left(C_{5}\right)$ and Amount of transport invoice $\left(C_{6}\right)$. Except the attributes $\left(C_{2}\right)$ and $\left(C_{3}\right)$, all others values take into account the movement in a period of 30 days. The variables $C_{1}, C_{3}$ and $C_{5}$ are called positive, ie, must be maximized. The Variables $C_{2}, C_{4}$ and $C_{6}$ represent cost and should be minimized. Customers are represented by $A_{n}$. The decision matrix for this problem is presented in Table 2 .

Table 2: Decision matrix

\begin{tabular}{crccccc}
\hline$N^{\mathbf{o}}$ & \multicolumn{1}{c}{$C_{1}$} & $C_{2}$ & $C_{3}$ & $C_{4}$ & $C_{5}$ & $C_{6}$ \\
\hline$A_{1}$ & $200.000,00$ & 15 & 79 & 100 & 5 & 85 \\
$A_{2}$ & $150.000,00$ & 0 & 97 & 70 & 3 & 89 \\
$A_{3}$ & $87.000,00$ & 2 & 77 & 23 & 7 & 75 \\
$A_{4}$ & $218.000,00$ & 23 & 60 & 89 & 12 & 100 \\
$A_{5}$ & $90.000,00$ & 8 & 34 & 34 & 9 & 90 \\
$A_{6}$ & $76.000,00$ & 10 & 65 & 50 & 5 & 54 \\
$A_{7}$ & $55.000,00$ & 4 & 84 & 39 & 2 & 76 \\
$A_{8}$ & $126.000,00$ & 0 & 100 & 61 & 14 & 120 \\
$A_{9}$ & $142.000,00$ & 3 & 43 & 70 & 12 & 160 \\
$A_{10}$ & $69.000,00$ & 2 & 71 & 44 & 8 & 87 \\
\hline
\end{tabular}

We used three different methods to solve the problem presented. The first was using Fuzzy Rules Based Systems. In the second method was applied the concepts of Fuzzy TOPSIS and, finally, in the third method, the Fuzzy FTOPSIS. The results obtained by these methods are presented in sequence.

\subsection{Fuzzy Rule Based System}

The Fuzzy Rules Based Systems have four main modules: a fuzzy rules base, a fuzzy inference engine, and fuzzification/defuzzification modules [14].

The existing rules in the rules base can be defined empirically or by a tool for automatic generation of rules from existing data. In this paper, the rules base was defined empirically by an expert.

The data to be analyzed by Fuzzy Rules Based System, presented in Table 2, were normalized by Equation (6). Specifically for Fuzzy Rules Based Systems, the Equation (7) can not be used to normalize the attributes $C_{2}, C_{4}$ and $C_{6}$ representing cost and should be minimized. This occours because, in the Fuzzy TOPSIS, the best input values (highest value for positive attributes and less value for negative attributes) have the input value 1 which, in domain $[0,1]$ in Fuzzy Rules Based Systems, represents the worst possible value as shown in Table 1.

The existing rules and the fuzzy sets defined for the variables, were defined by the expert. Due to the large number of possible rules, the rules base was defined based on the sample of data analyzed. Thus, for the problem presented in Table 2, 171 rules were defined empirically by the expert. The method of inference used was Mamdani [15] with the defuzzification by the Center of Area. Within this context, the membership function of the customer (consequent of the rule) used by the expert is equivalent to that shown in Table 1. At the end of the process of defuzzification, the customer with the final result closer to 1 will be classified as the best among those examined.

According to the rules base, the Fuzzy Rules Based System classified the customers as follows: $A_{2}>A_{1}>A_{6}>$ $A_{8}>A_{7}>A_{3}>A_{4}>A_{10}>A_{5}>A_{9}$. Thus, according to the rules base and the definitions of fuzzy variables made by the expert, the best customer is the Customer 2 and the worst customer is the Customer 9. The results of inference for all cases can be found in Table 4 .

\subsection{Fuzzy TOPSIS}

To work with the data in the Fuzzy TOPSIS, we must normalize them through Equations (6) and (7) as described in section 3. In the case study using the Fuzzy TOPSIS, greater importance was assigned to the variable Revenue $\left(C_{1}\right)$, weight 2 (Low). To the other variables involved in the problem was assigned weight 1 (Very Low). The process of fuzzification of the data is performed based on the membership function shown in Equation (1) associated with the data of Table 1 . The weight of the attributes uses the equivalence presented in the same table. 
The weighted matrix that takes into account the fuzzy sets of attributes with their respective weights is defined by Equation (2). From the data of fuzzy weighted matrix, the distance of each customer $A_{n}$ to FPIS and FNIS can be calculated by Equations (8) and (9), respectively. The degree of similarity with the FPIS to each customer $A_{n}$ is defined by Equation (10).

According to the preferences given, the Fuzzy TOPSIS classified the customers as follows: $A_{2}>A_{1}>A_{3}>A_{6}>$ $A_{7}>A_{8}>A_{5}>A_{4}>A_{10}>A_{9}$. Thus, the best customer is the Customer 2 and the worst customer is the Customer 9. The Table 4 presents the results obtained by the Fuzzy TOPSIS for all customers.

\subsection{Fuzzy F-TOPSIS}

To solve the proposed problem using the Fuzzy F-TOPSIS two fuzzy decision matrices were generated, in addition to add the attribute $C_{n+1}$ with the results of inference based on the rules defined by the expert. Thus, we improve the ability of treatment of vagueness in the Fuzzy TOPSIS through the combination of the mathematical process presented in the original method, with the empirical knowledge of an expert in decision making process. The new decision matrix is presented in Table 3.

Table 3: Decision matrix with the attribute $C_{n+1}$

\begin{tabular}{cccccccc}
\hline$N^{\mathbf{o}}$ & $C_{1}$ & $C_{2}$ & $C_{3}$ & $C_{4}$ & $C_{5}$ & $C_{6}$ & $C_{7}$ \\
\hline$A_{1}$ & $200.000,00$ & 15 & 79 & 100 & 5 & 85 & 0,5618 \\
$A_{2}$ & $150.000,00$ & 0 & 97 & 70 & 3 & 89 & 0,6250 \\
$A_{3}$ & $87.000,00$ & 2 & 77 & 23 & 7 & 75 & 0,4057 \\
$A_{4}$ & $218.000,00$ & 23 & 60 & 89 & 12 & 100 & 0,3899 \\
$A_{5}$ & $90.000,00$ & 8 & 34 & 34 & 9 & 90 & 0,2863 \\
$A_{6}$ & $76.000,00$ & 10 & 65 & 50 & 5 & 54 & 0,5010 \\
$A_{7}$ & $55.000,00$ & 4 & 84 & 39 & 2 & 76 & 0,4128 \\
$A_{8}$ & $126.000,00$ & 0 & 100 & 61 & 14 & 120 & 0,4132 \\
$A_{9}$ & $142.000,00$ & 3 & 43 & 70 & 12 & 160 & 0,2500 \\
$A_{10}$ & $69.000,00$ & 2 & 71 & 44 & 8 & 87 & 0,3245 \\
\hline
\end{tabular}

The attribute $C_{7}$ should be maximized, therefore, should be normalized by Equation (6). From the normalized values will be generated two matrices. The first matrix $V_{1}$ will be generated by same process of Fuzzy TOPSIS, however, the matrix $V_{2}$ will be assembled by fuzzy sets with the lowest membership function for $r_{i j}$.

According to Table 1 , to allow the attribute $C_{n+1}$ has a greater importance degree in relation to others, by simply assign greater weight to him than to other attributes. The reverse is also true. Thus, in order to give greater importance to attribute $C_{7}$, its importance degree will be 5 (Very High). Other attributes remain with the weights assigned in section 5.2.

Like the Fuzzy TOPSIS, presented in section 3, we also performed the calculation of the weighted matrix $V_{1}$ and $V_{2}$ by Equation (2). Calculated weighted matrix $V_{1}$ and $V_{2}$, the definition of FPIS and FNIS will be made as presented in Equations (11) and (12), respectively. According to the expert involved in the project, the results obtained by this process were more satisfactory than others, because the empiric knowledge has been considered in the analysis.

Defined FPIS and FNIS as mentioned in section 4, the calculation to determine the distance from the customer $A_{n}$ to FPIS and FNIS is done by Equations (13) and (14), respectively. The set $\tilde{v} 1_{i j}$, in the matrix $V_{1}$, has its influence level $I L_{\tilde{v} 1_{i j}}$ defined by Equation (11) and the set $\tilde{v} 2_{i j}$, in the matrix $V_{2}$, has its influence level $\left(I L_{\tilde{v} 2_{i j}}\right)$ defined by Equation (12).

After the execution of described steps, taking into account the attribute $C_{7}$ and the two fuzzy sets with greater relevance for each $r_{i j}$, the Fuzzy F-TOPSIS classified the customers as follows: $A_{2}>A_{1}>A_{6}>A_{5}>A_{3}>A_{10}>$ $A_{7}>A_{4}>A_{8}>A_{9}$. Thus, according to the Fuzzy F-TOPSIS method and the features listed, the best customer is the Customer 2 and the worst customer is the Customer 9. The complete classification is presented in Table 4.

\subsection{Analysis of Results}

After three different analysis of the data presented in Table 2, the results are compared in Table 4 that presents the customers and scores for each method. 
Table 4: Confrontation of results

\begin{tabular}{lllllll}
\hline \multicolumn{2}{c}{ SBRF } & \multicolumn{2}{c}{ Method } \\
\hline Cuzzy TOPSIS & \multicolumn{2}{c}{ Fuzzy F-TOPSIS } \\
\hline Customer & Score & Customer & Score & \multicolumn{1}{c}{ Customer } & Score \\
\hline Customer 2 & 0.6250 & Customer 2 & 0,1427 & Customer 2 & 0,8223 \\
Customer 1 & 0.5618 & Customer 1 & 0,1359 & Customer 1 & 0,8142 \\
Customer 6 & 0.5010 & Customer 3 & 0,1308 & Customer 6 & 0,6644 \\
Customer 8 & 0.4132 & Customer 6 & 0,1255 & Customer 5 & 0,6616 \\
Customer 7 & 0.4128 & Customer 7 & 0,1245 & Customer 3 & 0,6587 \\
Customer 3 & 0.4057 & Customer 8 & 0,1215 & Customer 10 & 0,5991 \\
Customer 4 & 0.3899 & Customer 5 & 0,1148 & Customer 7 & 0,5851 \\
Customer 10 & 0.3245 & Customer 4 & 0,1145 & Customer 4 & 0,5424 \\
Customer 5 & 0.2863 & Customer 10 & 0,1138 & Customer 8 & 0,5290 \\
Customer 9 & 0.2500 & Customer 9 & 0,1053 & Customer 9 & 0,4716 \\
\hline
\end{tabular}

The three methods classified the Customer 2 as first and the Customer 1 as second in the ranking. The difference begins from the third position in the ranking. In case of Customer 6, their best result in the Fuzzy F-TOPSIS when compared with the classical Fuzzy TOPSIS, was due to the influence of the results of Fuzzy Rules Based System. However, the same does not happen with other customers as, for example, the Customer 8. This happened because, in addition to considering the two fuzzy sets of greater pertinence in the process of Fuzzy F-TOPSIS, there is also the question of definition of FPIS and FNIS. If defined as presented in section 3, the FPIS and FNIS would not represent faithfully the best and the worst solution to the problem within the real context. Thus, when we use the values of the fuzzy sets corresponding to the input data, the definition of FPIs and FNIS are more appropriate to the context in which the problem is embedded. In general, this allowed a better analysis of results by experts.

\section{Conclusions}

The processes of decision making can take benefit from combination of theoretical and empirical knowledge of methods for the treatment of imprecision, not only in the data representation, but also in the moment of decision making. In this article, it was proposed Fuzzy F-TOPSIS method in order to explore the possibilities of flexibility of Fuzzy TOPSIS method, with the aim of attend this need by, merging the optimization technique already known in the literature with Fuzzy Rules Based System. Thus, the results presented in this article, beyond attend the initial expectations, they also make clear the viability of using the method in many areas of decision support that brings, among other advantages, the possibility of incorporate to decision making process the knowledge of experts, which can be expressed more appropriately in the form of rules. Among the future work, we would like to perform experiments to adapt the method Fuzzy F-TOPS to the process of group decision making as well as investigating techniques for automatic generation SBRF from data sets. The experiments to adapt the Fuzzy F-TOPSIS method to the group decision making as well as investigating techniques for automatic generation of rule base, are among the future work planned.

\section{References}

[1] Hwang C.L. \& Yoon K.: Multiple Attribute Decision Making: Methods and Application, Springer, New York (1981).

[2] Yang, T. \& Chou P.: Solving a multi-response simulation-optimization problem with discrete variables using a multipleattribute decision making method. Math Comput Simulat 68, 9-21, (2005).

[3] Yoon, K. \& Hwang, C.: Multiple attribute decision making. Thousand Oaks, CA, Sage Publication, (1995).

[4] Yang, T. \& Hung, C.: Multiple-attribute decision making methods for plant layout design problem. Robotics and ComputerIntegrated Manufacturing 23, 126-137, (2005).

[5] Salehi, M. \& Tavakkoli-Moghaddam, R.: Project Selection by Using a Fuzzy TOPSIS Technique. International Journal of Computer, Information, and Systems Science, and Engineering 2;2, Spring, 99-104, (2008).

[6] Pedrycz, W. \& Gomide, F.: An introduction to fuzzy sets: analysis and design, MIT Press, (1998).

[7] Chen, C. T.: Extensions of the TOPSIS for group decision making under fuzzy environment. Fuzzy Sets and Systems, 114, $1-9,(2000)$.

[8] Bellman, R. E., \& Zadeh, L. A.: Decision-making in a fuzzy environment management. Science, 17(4), 141-164, (1970).

[9] Saaty, T.L.: The Analytic Hierarchy Process, McGraw-Hill New York, (1980).

[10] Babic, Z. \& Plazibat N.: Ranking of enterprises based on multicriteria analysis, International Journal of Production Economics, 56-57, 29-35, (1998).

[11] Saaty, T.L.: Decision Making with Dependence and Feedback: The Analytic Network Process, Pittsburgh, RWS Publications, (1996). 
[12] Sarkis, J. \& Talluri, S.: A model for strategic supplier selection. In: Leenders, M. (Ed.), Proceedings of the 9th international IPSERA Conference. Richard Ivey Business School, London, Ontario, 652 - 661, (2000).

[13] Sun, C. \& Lin, G.T. R.: Using fuzzy TOPSIS method for evaluating the competitive advantages of shopping websites, Expert Systems with Applications, 36, 11764-11771, (2009).

[14] Klir, G. J. \& Yuan, B.: Fuzzy Sets and Fuzzy Logic: theory and applications, Prentice-Hall, (1995).

[15] Mamdani, E. H.: Applications of fuzzy algorithm for control of simple dynamic plant, Proceeding of the IEEE, v. 121, 1585-1588, (1974). 